Rev. Elev. Méd. vét. Pays trop., 1978, 31 (4) : 427-430.

\title{
La mammomonogamose des zébus en empire centrafricain
}

\author{
par J. VERCRUYSSE (*)
}

\begin{abstract}
RESUMÉ
Mammomonogamus nasicola occasionne une parasitose des voies respiratoires supérieures qui n'avait pas été signalée en E. C. A. Lors de 1062 autopsies effectuées à Bourar (E. C. A.) sur des zébus, l'auteur trouve 34 p. 100 d'animaux infestés par ce Nématode.

Le degré moyen d'infestation est de 2 à 3 couples par animal.

L'auteur donne des renseignements sur les lésions observées, leur localisation et le diagnostic différentiel.

Il situe l'importance économique de cette parasitose et évoque sa répartition géographique en Afrique.
\end{abstract}

\section{INTRODUCTION}

La mammomonogamose est une affection parasitaire due à la présence, au niveau de la partie supérieure de l'appareil respiratoire, de nématodes appartenant au sous-ordre des Strongyloidea, famille des Syngamidés, sousfamille des Syngaminés, genre Mammomonogamus Ryzhikov, 1948.

De janvier à août 1978 nous avons recherché, à Bouar, la présence de syngames chez les zébus (races Mbororo, Foulbé et Goudalé) de la partie occidentale de l'Empire Centrafricain (E. C. A.).

La région étudiée est du type soudano-guinéen. La saison des pluies dure sept mois, de mars à septembre, et la saison sèche cinq mois. Les zébus sont gardés par les Peuls qui pratiquent la transhumance en saison sèche.

(*) Adresse actuelle: Kempische Veldweg 94, 2230 Schilde, Belgique.

\section{MATÉRIEL ET MÉTHODES}

Pendant 8 mois consécutifs, dans un rayon de $50 \mathrm{~km}$ autour de Bouar, nous avons examiné 1062 zébus, spécialement au niveau du pharynx, du larynx et de la trachée. La recherche des syngames dans les cavités nasales a été effectuée chez une dizaine de sujets.

La mise en évidence des vers est facilitée par leur couleur rouge, leur situation superficielle sur la muqueuse et leur aspect en $Y$ dû au fait que les parasites adultes, un mâle et une femelle, vivent en accouplement permanent.

Pour identifier l'espèce, les vers sont éclaircis au lactophénol d'Amman (24-48 h) et observés au microscope. Pour la préparation définitive, les vers sont montés dans la gomme au chloral (2).

L'identification est effectuée par l'observation et la mensuration des caractères morphologiques déterminés selon MEJIA-GARCIA (6) : la structure interne de la capsule buccale, no- 
tamment le nombre des côtes chitineuses atteignant le bord de l'ouverture buccale ; l'aspect morphologique des glandes asophagiennes et la longueur de la queue de la femelle.

\section{RÉSULTATS}

\section{Espèce observée}

L'examen microscopique de 100 couples de vers a permis de constater qu'ils appartenaient tous à l'espèce Mammomonogamus nasicola Von Linstow, 1899. En effet, les côtes de la capsule buccale sont de longueur inégale; 1 à 3 seulement dépassent la moitié de la hauteur de celle-ci, les autres sont plus petites et plus courtes. Les glandes cesophagiennes sont digitiformes. La queue de la femelle est effilée et striée; et sa longueur moyenne (30 exemplaires examinés) est de $263 \mu$. Cette valeur diffère légèrement de celle trouvée par MEJIAGARCIA : $288 \pm 7 u$.

\section{Localisation des parasites}

Les parasites se localisent sur la muqueuse du bord postérieur de l'épiglotte, sur celle des ventricules latéraux et plus rarement sur les cordes vocales et jamais sur la muqueuse de la trachée ou des cavités nasales.

\section{Leur nombre}

Le plus souvent 2 à 3 couples sont trouvés sur la muqueuse mais des nombres plus élevés ne sont pas rares; chez un animal de 10 ans, on a trouvé 58 couples et chez d'autres respectivement $27,26,19,16$ et 12 couples. La moyenne sur 1062 animạu fut de 2,8 (tabl. 1).

\section{Réceptivité selon l'âge et le sexe}

On n'a pas pu établir de différence selon le sexe de l'animal. Quant à l'âge, le plus jeune animal avait 2 ans, la plupart avaient de 5 à 10 ans. Il s'agit donc d'une parasitose des adultes.

\section{Lésions macroscopiques}

Les lésions le plus fréquemment observées sont de petits ulcères ponctiformes correspondant aux points de fixation successifs des nématodes. Les nématodes baignent dans un abondant mucus spumeux.

\section{Incidence}

Au cours de l'année 1978, de janvier à septembre, le nombre de bêtes examinées variait mensuellement de 60 à 213 animaux (tabl. I).

\section{DISCUSSION}

La présence de $M$. nasicola est enregistrée chez les zébus en E. C. A. Il est surprenant que cette parasitose qui atteint 34 p. 100 des zébus n'ait jamais été signalée auparavant dans cette région. La présence de $M$. nasicola en Afrique est connue depuis le début du siècle grâce à la description originale de VON LINSTOW, 1899, à partir d'exemplaires prélevés dans les cavités nasales d'une chèvre autopsiée à Yaoundé (Cameroun). Un couple de syngames a été décrit en 1972 par GRABER et coll. chez Syncerus caffer dans l'est de l'E. C. A. et le nématode a été rapporté à $M$. nasicola. La mammomonogamose doit également exister au Tchad (5), car, dans un petit nombre de cas, des ceufs de syngames à deux blastomères ont été mis en

TABL. $\mathrm{N}^{\circ} \mathrm{I}$-Fréquence de Mammomonogamus nasicolia en E.C.A.

\begin{tabular}{|l|c|c|c|c|c|c|c|c|c|}
\hline \multirow{2}{*}{$\begin{array}{l}\text { Nombre de bêtes } \\
\text { exraminées }\end{array}$} & Janvier & Février & Mars & Avril & Mai & Juin & Juillet & Août & Total \\
\cline { 2 - 11 } & 93 & 60 & 93 & 146 & 155 & 171 & 131 & 213 & 1062 \\
\hline $\begin{array}{l}\text { Nombre de bêtes } \\
\text { positives }\end{array}$ & 26 & 19 & 34 & 64 & 69 & 66 & 48 & 38 & 364 \\
\hline Pourcentage & 28 & 32 & 36 & 44 & 44 & 39 & 40 & 17 & 34 \\
\hline Nombre de paires & 81 & 32 & 74 & 235 & 138 & 205 & 142 & 61 & 1008 \\
\hline Moyenne par bête & 3,1 & 1,7 & 2,2 & 3,7 & 2,6 & 3,1 & 2,9 & 1,6 & 2,8 \\
\hline
\end{tabular}


évidence lors d'examens coprologiques effectués sur des jeunes zébus. Faute d'autopsie, l'espèce en cause n'a jamais pu être précisée. Durant la même période, tous les moutons et les chèvres abattus dans la même région et examinés furent négatifs pour la syngamose.

La mammomonogamose en Asie et en Amérique a fait l'objet de plusieurs observations $(1,6,7)$.

En ce qui concerne le degré d'infestation parasitaire, le nombre de couples de parasites infestant l'animal est 2 à 3 (moyenne 2,8) et le plus élevé est 58. BUCKLEY (1) signale un cas d'un ovin porteur de 76 couples dans le pharynx, la trachée et les cavités nasales. PATNAIK (7) signale le cas d'un buffle hébergeant 68 couples. SACHS et collab. (9) trouvent 40 couples de Mammomonogamus sp. chez un ruminant sauvage.

Les lésions provoquées par le parasite sont caractérisées par la congestion de la muqueuse, par la présence de multiples pétéchies et exceptionnellement par des ulcères. Certains auteurs $(8 ; 10)$, observent des quintes de toux plus ou moins violentes. GRABER (communication personnelle) pense que les fausses réactions positives à la tuberculine ou à l'antigène péripneumonique, observées jadis sur des animaux atteints de toux et ne présentant à l'autopsie aucune lésion imputable à ces deux maladies, pourraient être dues à la mammomonogamose. Le problème mériterait d'être réétudié dans le centre et le sud du Cameroun où des cas de "Parasitoses pulmonaires " d'origine indéterminée chez les petits ruminants pourraient être dus à Mammomonogamus nasicola, ce parasite ayant été signalé chez des bovins au cours de ces dernières années (3).
Le diagnostic ante mortem par examen coprologique est malaisé. Les œufs de Mammomonogamus sont ellipsoïdes (76-98 « sur 36$60 \mu$ ) à coque épaisse et contiennent en général deux blastomères. Ils peuvent être confondus avec des œufs de strongles, notamment avec Bunostomum phlebotomum. L'examen coprologique de 200 zébus n'a révélé que 1 p. 100 de cas positifs avec des valeurs quantitatives très faibles.

Jusqu'ici, tous les essais pour tenter d'établir le cycle évolutif du ver sont demeurés infructueux (3). Au mois d'août, on observe un niveau d'infestation beaucoup plus bas que durant les autres mois. Il semble qu'un cycle direct puisse être exclu du fait de l'absence de corrélation entre le taux d'infestation et la saison des pluies.

Les symptômes discrets et le diagnostic laborieux sur l'animal vivant peuvent expliquer pourquoi la mammomonogamose est une parasitose peu connue en Afrique, d'autant plus que l'importance économique est difficile à évaluer. En E. C. A. cependant, 34 p. 100 des bovins adultes sont parasités par Mammomonogamus nasicola. Cette maladie est donc plus répandue qu'il ne semblait au premier abord.

L'homme peut héberger $M$. laryngeus ou $M$. nasicola et plusieurs cas ont été signalés en Amérique et en Asie. Jusqu'à présent, $M$. nasicola n'a jamais été signalé chez l'homme en Afrique.

\section{REMERCIEMENTS}

L'auteur tient à remercier vivement le $\mathrm{Dr}$ Graber qui a bien voulu aider à la détermination des Mammomonogamus.

\section{SUMMARY}

\section{Syngamosis of zebu cattle in central african empire}

From January to August 1978, 1062 post mortem examinations were carried out in Zebu cattle from west area (Bouar) in Centrafrican Empire. Mammomonogamus nasicola occurs in 34 p. 100 of cattle.

Each animal was found to be infected with 2 or 3 pairs.

The author points out briefly informations on localisations, diagnosis and lesions caused by these parasites.

Economic importance and geographical distribution of bovine syngamosis in Africa are discussed.

\section{RESUMEN}

\section{La mammomonogamosis de los cebues en Imperio} Centro-africano

Mammomonogamus nasicola provoca una parasitosis de las vias respiratorias superiores que no se habia señalado en Imperio Centroafricano. Durante 
1062 autopsias efectuadas en Buar (I. C. A.) en cebues, el autor encuentra 34 p. 100 de animales infestados por dicho nemátodo.

El grado medio de infestación es de 2 a 3 parejas por animal.

El autor da informes sobre las lesiones observadas, su localización y el diagnóstico diferencial.

Situa la importancia económica de este parasitosis y evoca su repartición geográfica en Africa.

\section{BIBLIOGRAPHIE}

1. BUCKLEY (J. J.). On Syngamus nasicola (Linstow, 1899) from sheep and cattle in the West Indies. $J$. Helminthol., 1934, $12: 47-62$.

2. EUZEBY (J.). Diagnostic expérimental des helminthoses animales. Paris, Vigot, 1958, $367 \mathrm{p}$.

3. EUZEBY (J.), GRABER (M.), GEVREY (J.), MEJIA (A.). Données récentes concernant la mammomonogamose en Amérique et aux Antilles. Bull. Acad. vét. Fr., 1977, $50: 267-273$.

4. GRABER (M.), EUZEBY (J.), GEVREY (J.), TRONCY (P. M.). Les Mammomonogamus des ruminants domestiques et sauvages. Annls. Parasit. hum. comp., 1972, $47: 225-241$.

5. GRABER (M.), EUZEBY (J.), GEVREY (J.), TRONCY (P. M.), THAL (J.). La mammomonogamose des ruminants domestiques et sauvages. Rev. Elev. Méd. vét. Pays trop., 1971, 24 : 525-541.

6. MEJIA-GARCIA (R. A.). Les mammomonoga- moses des ruminants domestiques et de l'homme au Mexique. Lyon, Service de Parasitologie de l'Ecole nationale vétérinaire de Lyon (France), 1978.

7. PATNAIK (M. M.). A note on bovine syngamosis. Indian vet. $J ., 1963,40: 272-274$.

8. ROSE-ROSETTE (R.). Un cas de syngamonose laryngien chez le mouton. Bull. Soc. Path. exot., $1934,28: 264-265$.

9. SACHS (R.), FRANK (H), BINDERNAGEL (J. A.). New host records for Mammomonogamus in African game animals through application of a simple method of collection. Vet. Rec., 1969, 5 : 562563.

10. TEUSCHER (E.), KOMIJIN (R. E.), ROBALINO (R.), SMITH (R, D.). Le diagnostic coprologique de la syngamose bovine en Equateur avec une note sur l'efficacité probable du tétramisole. Zentbl. Vet. Med. Reihe B., 1970, 17 : 324-330. 